Proc. XIX International School of Semiconducting Compounds, Jaszowiec 1990

\title{
THE INFLUENCE OF JAHN-TELLER EFFECT ON MAGNETIC PROPERTIES OF Fe-BASED SEMIMAGNETIC SEMICONDUCTORS
}

\author{
K. LEBECKI, A. TWARDoWsKI \\ Institute of Experimental Physics, Warsaw University, Hoża 69, 00-681 Warszawa, \\ Poland
}

AND Z. LIRo

High Pressure Center "Unipress", Sokolowska 29, 01-142 Warszawa, Poland (Received August 8, 1990)

\begin{abstract}
We report results of calculations of magnetic properties (specific heat, magnetization) of Fe-based Semimagnetic Semiconductors including Jahn-Teller coupling between $\mathrm{Fe}$ electronic states and phonons.
\end{abstract}

PACS numbers: 75.50.Pp, 71.70.Ch, 71.70.Ej, 75.90.+w

Iron based Semimagnetic Semiconductors (SMSC) [1] have been recently intensively studied $[2,3]$. Some experimental results suggest important role of Jahn-Teller (JT) coupling between Fe electronic states and phonons [3-5]. In this respect we extended calculation of Vallin [6] and studied in some detail influence of JT effect on specific heat and magnetization of Fe-type SMSC.

The total Hamiltonian is assumed to be in the form [6]:

$$
H=H_{\mathrm{cf}}+H_{\mathrm{so}}+H_{\mathrm{B}}+H_{\mathrm{vib}}+H_{\mathrm{JT}}
$$

where $H_{\text {cf }}$ describes crystal field, $H_{\text {so }}=\lambda L S$ is spin-orbit term, $H_{B}$ is magnetic field term, $H_{\text {vib }}=(1 / 2 \mu)\left(P_{u}^{2}+P_{v}^{2}+\mu^{2} \omega^{2}\left(Q_{u}^{2}+Q_{v}^{2}\right)\right)$ is vibrational term describing TA phonons of frequency $\omega[6]$ and $H_{\mathrm{JT}}=A\left(U_{u} Q_{u}+U_{v} Q_{v}\right)$ describes JT coupling with vibrational $\epsilon$ mode [6] (we used coupling constant $\left.V=A /(\mu \omega / \hbar)^{1 / 2}\right)$. For the basis wave function set we used electron-vibronic eigenfunctions of $H_{\mathrm{cf}}+H_{\mathrm{so}}+H_{\mathrm{vib}}$. Six vibrational states were included into basis. We assumed that $H_{\mathrm{cf}}$ and $H_{\mathrm{so}}$ split ${ }^{5} E$ ground term of $\mathrm{Fe}^{++}$ion into five equally spaced levels $\left(\Delta E=6 \lambda^{2} / 10 D q+\right.$ $\rho$, where $D q$ is the crystal field parameter [6]). This assumption is equivalent 
to neglecting admixture of excited ${ }^{5} T_{2}$ term wavefunctions into ${ }^{5} E$ ground term wavefunctions [6,7]. Consequently, orbital angular momentum $\boldsymbol{L}$ has only zero matrix elements within ${ }^{5} E$ ground term. Therefore $H_{B}$ is taken in an extended form [7] simulating ${ }^{5} E-{ }^{5} T_{2}$ mixing.

Hamiltonian (1) is solved numerically by diagonalization of $60 \times 60$ matrix. JT coupling results in downward shift of the ground state and reduction of the energy gap between the ground and the first excited states. The obtained energy levels were subsequently used for calculation of magnetization and specific heat.

In Fig. 1 we display magnetization calculated for different magnetic field orientations for $V=15 \mathrm{~cm}^{-1}$ as well as for $V=0$ (no JT coupling). We notice that

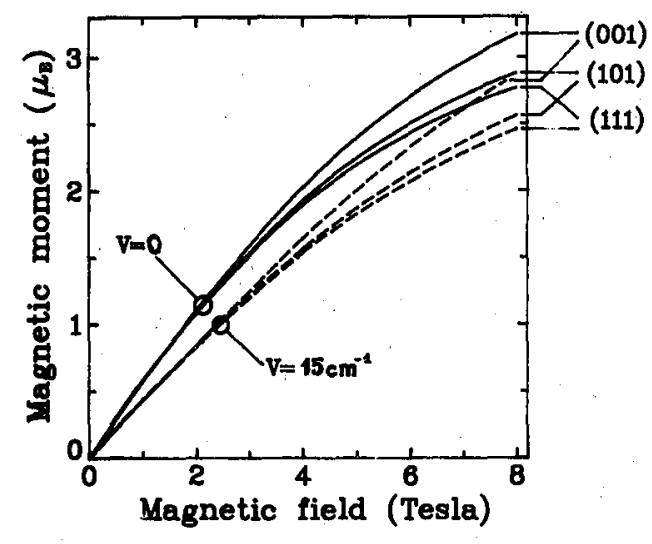

Fig. 1. Magnetization (in Bohr magnetons) as a function of magnetic field, oriented paralelly to (001), (101), (111); solid lines: $V=0$ i.e. no JT coupling $\left(\lambda=-66.8 \mathrm{~cm}^{-1}\right)$; dashed lines: $V=15 \mathrm{~cm}^{-1}\left(\lambda=-90.7 \mathrm{~cm}^{-1}\right)$; energy of coupled phonon $\hbar \omega=20 \mathrm{~cm}^{-1}$, $D q=-257 \mathrm{~cm}^{-1}, \varrho=0.95 \mathrm{~cm}^{-1}$. (Parameters $D q, \lambda, \varrho$ were chosen to provide energy difference $E_{T 1}-E_{A 1}=16 \mathrm{~cm}^{-1}$ the value close to the experimental observation for $\mathrm{CdFeTe}, \mathrm{CdFeSe}, \mathrm{ZnFeSe})$.

we used different parameter set for $V=0$ and for non zero $V$ to obtain the same energy separation between the two lowest states. In this case field induced mixing between singlet ground state and magnetically active excited states is reduced for non zero $V$ and then magnetization is smaller. This effect increases with increase of JT coupling (parameter $V$ ) and decrease of the coupled phonon frequency $\omega$. JT coupling does not change magnetization anisotropy significantly.

Magnetic contribution to the specific heat $\left(C_{m}\right)$ was evaluated in a way similar to the usual experimental method [4]:

$$
C_{m}(T, B)=C_{p}-C_{\text {latt }}
$$

where $C_{p}$ is heat capacity resulting from electron-vibronic levels and $C_{\text {latt }}$ is specific heat arising from lattice TA phonons. The example results are shown in Fig. 2, where we also illustrated the effect of difference between local $\hbar \omega_{\text {loc }}$ TA phonon 
(which couples to the electronic Fe ion states) and lattice TA phonon. We notice rather slight influence of $\mathrm{JT}$ coupling on $C_{m}$. On the other hand the absolute value

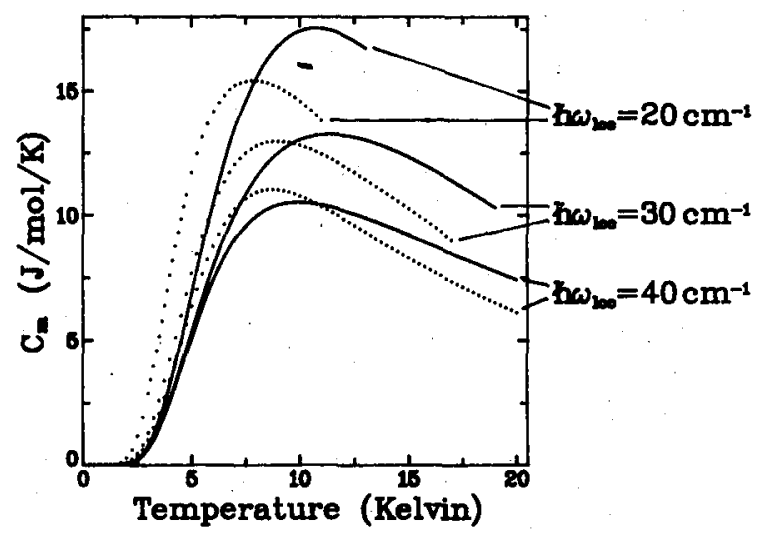

Fig. 2. Magnetic contribution to specific heat $C_{m}$ as a function of temperature. Solid lines indicate no JT coupling and dotted lines indicate $V=15 \mathrm{~cm}^{-1}$. Energy of lattice phonon $\hbar \omega_{\text {latt }}$ is set to $40 \mathrm{~cm}^{-1}$ and local phonons $\hbar \omega_{\text {loc }}=20,30,40 \mathrm{~cm}^{-1}, \lambda=$ $-66.8 \mathrm{~cm}^{-1}, D q=-257 \mathrm{~cm}^{-1}, \varrho=0.95 \mathrm{~cm}^{-1}$. (Parameters $D q, \lambda, \varrho$ were chosen to provide energy difference $E_{T 1}-E_{A 1}=16 \mathrm{~cm}^{-1}$ the value close to the experimental observation for CdFeTe, CdFeSe, ZnFeSe).

of $C_{m}$ increases strongly with decreasing energy of coupled phonon. Therefore we believe that exceptionally large $C_{m}$ of CdFeSe [4] results rather from disregarding local phonons in $C_{\text {latt }}$ than JT effect.

In conclusion we found rather small influence of JT coupling on magnetic properties of Fe-type SMSC. Experimental data for the systems with probably strong JT effect (CdFeTe, CdFeSe) are too poor to provide quantitative comparison with the calculations.

\section{References}

[1] J.K. Furdyna, J. Kossut (eds), Diluted Magnetic Semiconductors, in Semiconductors and Semimetals, Vol. 25, Academic Press, San Diego 1988;

J.K. Furdyna, J. Appl. Phys. 64, R29 (1988).

[2] A. Mycielski, J. Appl. Phys. 63, 3279 (1988).

[3] A. Twardowski, J. Appl. Phys. 67, 5108 (1990).

[4] A. Twardowski, H.J.M. Swagten, W.J.M. de Jonge, Phys. Rev. B 42, 2455 (1990).

[5] G.A. Slack, S. Roberts, J. Vallin, Phys. Rev. 187, 511 (1969).

[6] J.T. Vallin, Phys. Rev. B 2, 2390 (1970).

[7] J.T. Vallin, G.A. Slack, C.C. Bradley, Phys. Rev. B 2, 4406 (1970). 